\title{
The potential benefit of levothyroxine treatment during pregnancy: another step forward
}

Tim I M Korevaar and Robin P Peeters

Departments of Internal Medicine and Academic Center for Thyroid Disease, Erasmus University Medical Center, the Netherlands
Correspondence should be addressed to T I M Korevaar

Email

t.korevaar@erasmusmc.n

Thyroid hypofunction during pregnancy is associated with a wide range of adverse pregnancy and child outcomes such as miscarriage, premature delivery, abnormal birth weight and suboptimal offspring neurodevelopment. Thyroid autoimmunity, reflected by thyroperoxidase antibody (TPOAb) positivity, is the most important risk factor for thyroid hypofunction. During pregnancy, TPOAb-positive women have higher TSH and lower FT4 concentrations and a higher risk of overt and subclinical hypothyroidism than TPOAb-negative women $(1,2)$. Intriguingly, TPOAb positivity is associated in several observational studies with a higher risk of adverse outcomes including miscarriage, premature delivery and gestational diabetes, even in euthyroid women $(2,3,4$, $5,6,7)$. Such studies suggest potential adverse effects of thyroid autoimmunity on pregnancy outcome. However, the mechanisms underlying these associations, as well as causality of the associations, remain to be elucidated. In this context, the study by Nazarpour et al. in the current issue of the European Journal of Endocrinology is a very valuable contribution.

Two main hypotheses have been proposed regarding the underlying mechanism: the first being that TPOAb positivity is more likely to occur in women with a higher susceptibility to autoimmune disease in general. A higher prevalence of (mild) autoimmune disease would cause the higher risk of adverse outcomes via non-thyroidal pathways. In other words, autoimmune disease is a confounder in the association of TPOAb positivity with adverse pregnancy outcomes. In this case, levothyroxine will not be a plausible treatment. The second hypothesis is that TPOAb positivity leads to (mild) thyroid hypofunction and that suboptimal thyroid hormone availability would subsequently increase the risk of adverse outcomes. In that case, restoring thyroid hormone availability may overcome the negative effects on pregnancy outcome.

www.eje-online.org DOI: 10.1530/EJE-16-1058
(C) 2017 European Society of Endocrinology Printed in Great Britain
During pregnancy, high concentrations of hCG stimulate the thyroid leading to a higher FT4 and a lower TSH (8). Recently, our group showed that in TPOAb-positive women, higher hCG concentrations are not associated with higher FT4 or lower TSH (9). This indicates that TPOAb-positive women have an impaired thyroidal response to hCG stimulation. We subsequently showed that out of all TPOAb-positive women, those with the lowest thyroidal response to hCG stimulation have an up to 2.8-fold higher risk of premature delivery, whereas women with a relatively normal hCG response do not have a higher risk $(9,10)$. This implies that TPOAb positivity is associated with adverse pregnancy outcomes via changes in thyroid function (or more specifically via the thyroidal response to hCG stimulation) instead of via other autoimmune processes. Still, it should be noted that these data come from observational studies and thus cannot infer causality. The only study design that can truly answer the question of causality is a study that includes (1) randomization: to overcome any confounding and (2) thyroid hormone supplementation: to restore the potential lack of thyroid hormone availability and confirm a thyroid hormone pathway.

In the current study by Nazarpour et al. (10), 1734 pregnant women (a necessary extension from the 1600 initially planned to adhere to their power calculation) were screened for thyroid dysfunction (measuring TSH and the FT4 index (FT4I)) and TPOAb positivity. This yielded 131 TPOAb-positive women without (subclinical) hypothyroidism or hyperthyroidism eligible for randomization. Four to eight days after randomization, women in the treatment group received levothyroxine based on their TSH at baseline $(<1 \mathrm{U} / \mathrm{L}: 0.5 \mu \mathrm{g} / \mathrm{kg} /$ day; 1-2U/L: $\quad 0.75 \mu \mathrm{g} / \mathrm{kg} /$ day; $>2 \mathrm{U} / \mathrm{L}$ and/or TPOAbs $>1500 \mathrm{IU} / \mathrm{mL}$ : $1 \mu \mathrm{g} / \mathrm{kg} /$ day). Although both the patient and the treating physicians were aware of what group the patient was randomized to, the physicians that

Published by Bioscientifica Ltd. 
determined the pregnancy outcome were blinded, making this a single-blinded randomized controlled trial.

In the treatment group, median TSH concentrations decreased from 3.7 to 1.5 and $1.0 \mathrm{U} / \mathrm{L}$, whereas TSH remained stable in the non-treated group at 3.2, 3.9 and $3.4 \mathrm{U} / \mathrm{L}$ during the first, second and third trimester respectively. Levothyroxine treatment reduced the risk of premature delivery by $70 \%(7.1 \%$ vs $23.7 \%$; RR: 0.30 (95\% CI: 0.1-0.85)). Levothyroxine treatment did not change the percentage of miscarriage (3.6\% vs $3.4 \%$ ) but due to the low number of cases (4/114 that completed follow-up) analyses on this outcome were considered to be underpowered.

The current study was a replication of a study published by Negro et al. in 2006 (11), in which 115 TPOAb-positive women were randomized to receive levothyroxine treatment using the same dosing regimen or remain untreated. The effect of levothyroxine treatment on the risk of premature delivery in TPOAb-positive women in the study by Nazarpour et al. (7.1\% vs $23.7 \%$ ) was almost an exact replication of the study by Negro et al. (22.4\% vs $7.0 \%)$ (11). Interestingly, the authors of the current study also stratified the analyses according to the TSH concentration at baseline, demonstrating that the main reduction in premature delivery was achieved in women with an initial TSH concentration of $\geq 4 \mathrm{U} / \mathrm{L}(<4 \mathrm{U} / \mathrm{L}$ : $5.6 \%$ reduction $(P=0.69)$, vs a $24.1 \%$ reduction for $\geq 4 \mathrm{U} / \mathrm{L}$ $(P=0.01))$. Even though this trial was not designed to detect such differences, these results are very much in line with recent observational studies that indicate that the risk of adverse pregnancy outcomes in TPOAb-positive women is dependent on their TSH concentration. Recent studies show that TPOAb-positive women with a high-normal TSH have a higher risk of miscarriage, premature delivery and gestational diabetes than TPOAb-positive women with low (normal) TSH concentrations. In addition, a recent randomized controlled trial has indicated that levothyroxine treatment in TPOAb-positive women with a TSH: $0.5-2.5 \mathrm{U} / \mathrm{L}$ did not lower the risk of miscarriage or premature delivery, although some important methodological issues hamper the interpretation of these results (12). Taken together, these data suggest that a higher TSH in TPOAb-positive women is likely to indicate a more severe form of autoimmunity, having more impact on thyroid functional capacity and the overall thyroid hormone availability during pregnancy.

In contrast to premature delivery, miscarriage is notoriously difficult to study given that roughly $80 \%$ of all miscarriages occur in the first trimester, and many women miscarry before they know that they are pregnant. In contrary to the current study, Negro et al. found that levothyroxine treatment reduced the rate of miscarriage (3.5\% vs $13.8 \%)$ (11). This could be because Negro et al. included women at an earlier gestational age than the current study (10.4 \pm 3 weeks vs $11.4 \pm 4$ weeks). In the study by Negro et al., the vast majority of miscarriages occurred during the first trimester, and the time between treatment initiation and miscarriage was very short (11). Interestingly, to overcome potential bias due to very early miscarriages or late inclusions, randomized controlled trials that include TPOAb-positive women before pregnancy are currently ongoing (TABLET trial, ISRCTN: 15948785 and T4LIFE trial, NTR3364).

In the current study, overt and subclinical hypothyroidism was defined by a normal FT4 with a TSH above $2.5 \mathrm{U} / \mathrm{L}$. Although it has become apparent that this cut-off is too low to define an abnormal TSH $(13,14$, $15,16)$, the use of this cut-off in the current study leads to a sensible control group of women with a very low risk of having or developing an abnormal thyroid function (e.g. TPOAb-negative women with a TSH of 0.1-2.5 U/L). Another strength of this study is the fact that FT4 was estimated by calculating a free thyroxine index, which is a more robust way of measuring FT4 concentrations given that FT4 assays may be flawed due to thyroid-binding protein interference, especially during late pregnancy.

The levothyroxine dosing strategy based on weight and TSH concentration at presentation mimics clinical practice and is also a strength of the current study. On the other hand, it may be that the lack of treatment effect in the women with a TSH $<4.0 \mathrm{U} / \mathrm{L}$ was because they were treated with a lower dose. In the face of two randomized controlled trials showing a benefit of levothyroxine in TPOAb-positive women, the next step could be to further study what the optimal dosage would be. Importantly, recent studies, as well as preliminary results of unpublished studies, have suggested that highnormal maternal thyroid function during pregnancy may be harmful for the fetus. Especially during early pregnancy, it would be a safe alternative to start with a low and fixed dose at presentation and titrate the dose, if necessary, after 4-6 weeks. We look forward to future trials that may incorporate risk stratification markers such as TSH, TPOAbs and/or hCG at presentation to further improve clinical treatment regimens of thyroid disease during pregnancy.

\section{Declaration of interest}

T I M K declared that he has received a lecture fee from Berlin-Chemie AG. $\mathrm{R} P \mathrm{P}$ declared that he has received lecture fees from GoodLife Fertility B.V. and Institut Biochimique SA (IBSA). 


\section{Funding}

This research did not receive any specific grant from any funding agency in the public, commercial or not-for-profit sector.

\section{References}

1 Medici M, de Rijke YB, Peeters RP, Visser W, de Muinck KeizerSchrama SM, Jaddoe VV, Hofman A, Hooijkaas H, Steegers EA, Tiemeier $\mathrm{H}$ et al. Maternal early pregnancy and newborn thyroid hormone parameters: the generation R study. Journal of Clinical Endocrinology and Metabolism 201297 646-652. (doi:10.1210/ jc.2011-2398)

2 Thangaratinam S, Tan A, Knox E, Kilby MD, Franklyn J \& Coomarasamy A. Association between thyroid autoantibodies and miscarriage and preterm birth: meta-analysis of evidence. BMJ 2011 342 d2616. (doi:10.1136/bmj.d2616)

3 He X, Wang P, Wang Z, He X, Xu D \& Wang B. Thyroid antibodies and risk of preterm delivery: a meta-analysis of prospective cohort studies. European Journal of Endocrinology 2012167 455-464. (doi:10.1530/EJE-12-0379)

4 Karakosta P, Alegakis D, Georgiou V, Roumeliotaki T, Fthenou E, Vassilaki M, Boumpas D, Castanas E, Kogevinas M \& Chatzi L. Thyroid dysfunction and autoantibodies in early pregnancy are associated with increased risk of gestational diabetes and adverse birth outcomes. Journal of Clinical Endocrinology and Metabolism 2012 97 4464-4472. (doi:10.1210/jc.2012-2540)

5 Liu H, Shan Z, Li C, Mao J, Xie X, Wang W, Fan C, Wang H, Zhang H, Han C et al. Maternal subclinical hypothyroidism, thyroid autoimmunity, and the risk of miscarriage: a prospective cohort study. Thyroid 201424 1642-1649. (doi:10.1089/thy.2014.0029)

6 Korevaar TI, Schalekamp-Timmermans S, de Rijke YB, Visser WE, Visser W, de Muinck Keizer-Schrama SM, Hofman A, Ross HA, Hooijkaas H, Tiemeier $\mathrm{H}$ et al. Hypothyroxinemia and TPO-antibody positivity are risk factors for premature delivery: the generation R study. Journal of Clinical Endocrinology and Metabolism 201398 4382-4390. (doi:10.1210/jc.2013-2855)

7 Ying H, Tang YP, Bao YR, Su XJ, Cai X, Li YH \& Wang DF. Maternal TSH level and TPOAb status in early pregnancy and their relationship to the risk of gestational diabetes mellitus. Endocrine $2016 \mathbf{5 4}$ 742-750. (doi:10.1007/s12020-016-1022-6)
8 Glinoer D, de Nayer P, Bourdoux P, Lemone M, Robyn C, van Steirteghem A, Kinthaert J \& Lejeune B. Regulation of maternal thyroid during pregnancy. Journal of Clinical Endocrinology and Metabolism 199071 276-287. (doi:10.1210/jcem-71-2-276)

9 Korevaar TI, Steegers EA, Pop VJ, Broeren MA, Chaker L, de Rijke YB, Jaddoe VW, Medici M, Visser TJ, Tiemeier H et al. Thyroid autoimmunity impairs the thyroidal response to hCG: two population-based prospective cohort studies. Journal of Clinical Endocrinology and Metabolism 2016 jc20162942. In Press (doi:10.1210/jc.2016-2942)

10 Nazarpour S, Ramezani Tehrani F, Simbar M, Tohidi M, Alavi Majd H $\&$ Azizi F. Effects of levothyroxine treatment on pregnancy outcomes in pregnant women with autoimmune thyroid disease. European Journal of Endocrinology 2016176 253-265. (doi:10.1530/EJE-16-0548)

11 Negro R, Formoso G, Mangieri T, Pezzarossa A, Dazzi D \& Hassan $\mathrm{H}$. Levothyroxine treatment in euthyroid pregnant women with autoimmune thyroid disease: effects on obstetrical complications. Journal of Clinical Endocrinology and Metabolism 200691 2587-2591. (doi:10.1210/jc.2005-1603)

12 Korevaar TI \& Peeters RP. Letter to the Editor: methodological comments on the study by Negro et al. entitled 'Impact of levothyroxine in miscarriage and preterm delivery rates in first trimester thyroid antibody-positive women with TSH $<2.5$ IU/L'. Journal of Clinical Endocrinology and Metabolism 2016101 L101-L102. (doi:10.1210/jc.2016-2989)

13 Korevaar TI, Medici M \& Peeters RP. Subclinical hypothyroidism overdiagnosis in pregnant women. JAMA Internal Medicine 2015175 1872-1873. (doi:10.1001/jamainternmed.2015.5550)

14 Li C, Shan Z, Mao J, Wang W, Xie X, Zhou W, Li C, Xu B, Bi L, Meng $T$ et al. Assessment of thyroid function during first-trimester pregnancy: what is the rational upper limit of serum TSH during the first trimester in Chinese pregnant women? Journal of Clinical Endocrinology and Metabolism 201499 73-79. (doi:10.1210/jc.20131674)

15 Maraka S, Ospina NM, O'Keeffe DT, Espinosa De Ycaza AE, Gionfriddo MR, Erwin PJ, Coddington CC 3rd, Stan MN, Murad MH \& Montori VM. Subclinical hypothyroidism in pregnancy: a systematic review and meta-analysis. Thyroid 201626 580-590. (doi:10.1089/thy.2015.0418)

16 Medici M, Korevaar TI, Visser WE, Visser TJ \& Peeters RP. Thyroid function in pregnancy: what is normal? Clinical Chemistry 201561 704-713. (doi:10.1373/clinchem.2014.236646)

Received 22 December 2016

Accepted 16 January 2017 